\title{
FABRICATION AND SUB-ASSEMBLY OF ELECTROSTATICALLY ACTUATED SILICON NITRIDE MICROSHUTTER ARRAYS
}

\author{
Lance H. Oh ${ }^{l}$, Mary J. Li ${ }^{2}$,Kyowon Kim ${ }^{3}$, Dan Kelly ${ }^{3}$, Alexander Kutyrev ${ }^{4}$, Samuel H. Moseley ${ }^{2}$, Nick P. Costen ${ }^{l}$, \\ and George Manos ${ }^{2}$ \\ ${ }^{1}$ SGT Inc., Maryland, USA \\ ${ }^{2}$ NASA/Goddard Space Flight Center, Maryland, USA \\ ${ }^{3}$ ASRC Federal Corp., Maryland, USA \\ ${ }^{4}$ University of Maryland, Maryland, USA
}

\begin{abstract}
We have developed a new microshutter array (MSA) subassembly. The MSA and a silicon substrate are flip-bonded together. The MSA has a new back side fabrication process to actuate the microshutters electrostatically, and the new silicon substrate has light shields. The microshutters with a pixel size of $100 \times 200 \mu \mathrm{m}^{2}$ are fabricated on silicon with thin silicon nitride membranes. The microshutters rotate $90^{\circ}$ on torsion bars. The selected microshutters are actuated, held, and addressed electrostatically by applying voltages on the electrodes the front and back sides of the microshutters. The substrate has the light shield to block lights around the microshutters. Also, electrical connections are made from the MSA to a controller board via the substrate.
\end{abstract}

\section{INTRODUCTION}

In the Goddard Space Flight Center, we have developed a new MSA sub-assembly. The sub-assembly consists of the MSA and a silicon substrate. The MSA has a new back side fabrication process to actuate the microshutters electrostatically, and the new silicon substrate has light shields.

In the past, a large MSA array size of $175 \times 384$ had been developed, fabricated, and installed in the James Webb Space Telescope (JWST) as multi-object aperture selectors $[1,2]$. The JWST MSAs were magnetically opened and electrostatically held open. The magnetic operation, however, involved moving magnets thus complicated actuation schemes and increased the payload. Instead, the electrostatic actuation is simple. Thus, the weight of the MSA assembly is far reduced.

The microshutters were made with silicon nitride membranes on silicon with a pixel size of $100 \times 200 \mu \mathrm{m}^{2}$ and rotate $90^{\circ}$ on torsion bars. Figure 1 shows a scanning electron microscope (SEM) image of the MSA; on the front side, microshutter blades are suspended on the $2 \mu \mathrm{m}$ wide torsion bars, the gap around the microshutters is 2 $\mu \mathrm{m}$ wide, aluminum is patterned to create electrodes, and molybdenum nitride is patterned on top of the microshutter blade. Also, the JWST microshutters had the light shields patterned with aluminum overhang around the openings as shown in Figure 2. The purpose of the light shield is to block light passing through the perimeters around the microshutters. The light shield step required 3 lithography steps: a sacrificial layer, partially exposed bumps pattern on the sacrificial layer, and an aluminum pattern over the sacrificial layer. In order to avoid these three lithography steps plus an aluminum deposition, we decided to develop a new silicon substrate with the light shields.

The silicon substrate was first developed and used as a mechanical support for the MSA and an electrical interface between the MSA and the external control electronics on a printed circuit board as shown in Figure 3. For the first time, we present the new MSA indium-flip-chip bonded to the silicon substrate with light shields that block light leak around the openings around the microshutters. The front side of the MSA is flip-chip bonded to the aluminum electrodes on the silicon oxide surface of the silicon substrate. The front electrodes are connected to the substrate electrodes via indium bumps while the back side electrodes are wire bonded to the substrate electrodes.

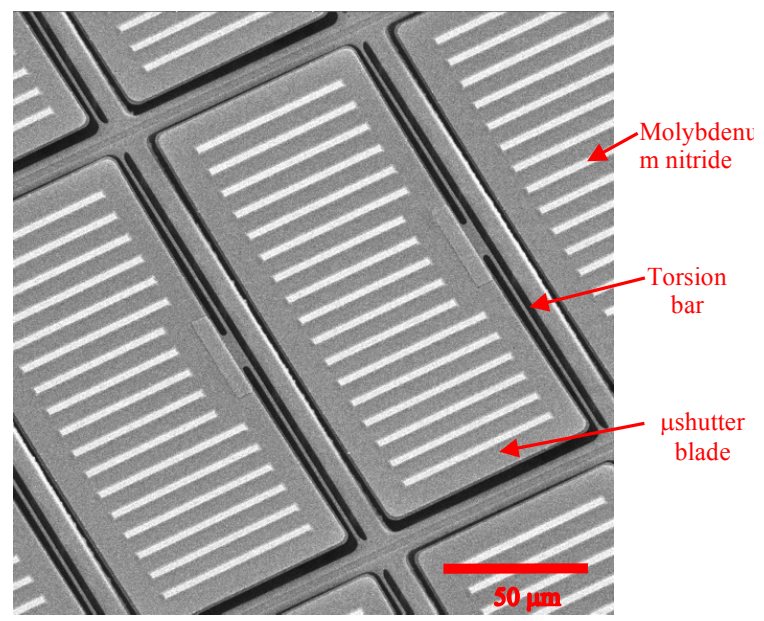

Figure 1: A SEM image of the front side image of the MSA. On the microshutters, the patterned microshutter blade, torsion bar and strips of molybdenum nitride are shown.

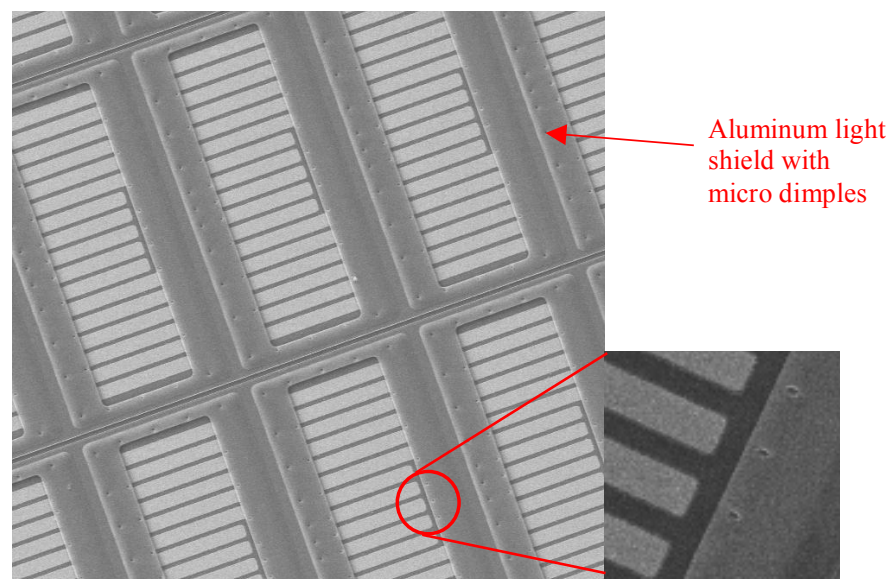

Figure 2: A SEM image of the MSA with aluminum light shields that prevent light passing through the perimeters around the microshutters. There are $1 \mu \mathrm{m}$ wide and $0.25 \mu \mathrm{m}$ deep dimples of the light shield bumps to prevent the stiction of the microshutters to the light shields. A close-up photo of the bumps is shown. 


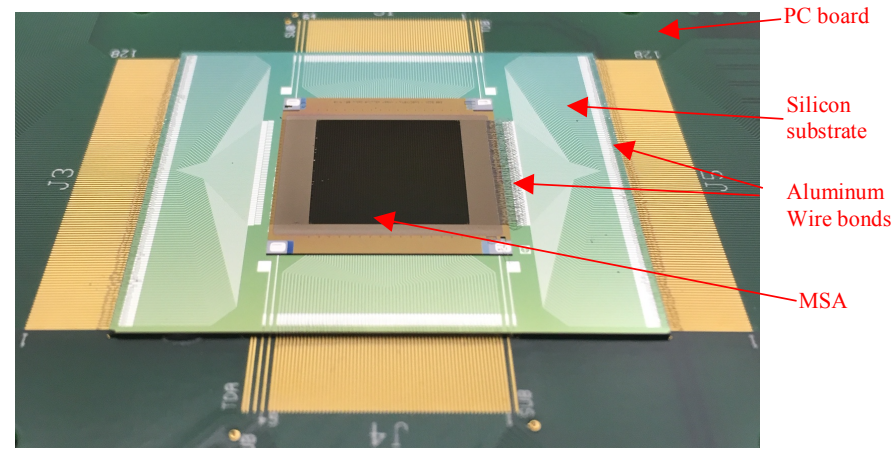

Figure 3: A photo of a sub-assembly of the MSA. The MSA is flipbonded to a silicon substrate with indium bumps. The front side of the MSA is flip-chip bonded to the substrate electrodes. Thus, the back side of the MSA is shown in the photo. The MSA and the substrate is glued to a PC board with an epoxy. Aluminum wirebonds are used to connect the back electrodes of the MSA to the substrate and the substrate electrodes to the PC board.

Lastly, in order to open a microshutter electrostatically, we applied a pulsed DC voltage on the front electrode and a DC voltage to the back electrode as shown in Figure 4. The front side electrodes run vertically and separated by $2 \mu \mathrm{m}$ gap in aluminum while the back side electrodes run horizontally. The back electrodes are individually separated by gaps in aluminum electrodes. Previously in the JWST MSA, we had used e-beam deposited alumina as an electrical insulator for the back electrodes. Even with $1 \mu \mathrm{m}$ thick ebeam alumina, the resistance and breakdown voltage were still low. Thus, we decided to deposit alumina in an atomic layer deposition (ALD) system. Incorporating the ALD alumina required a development of a new fabrication process on the back side.

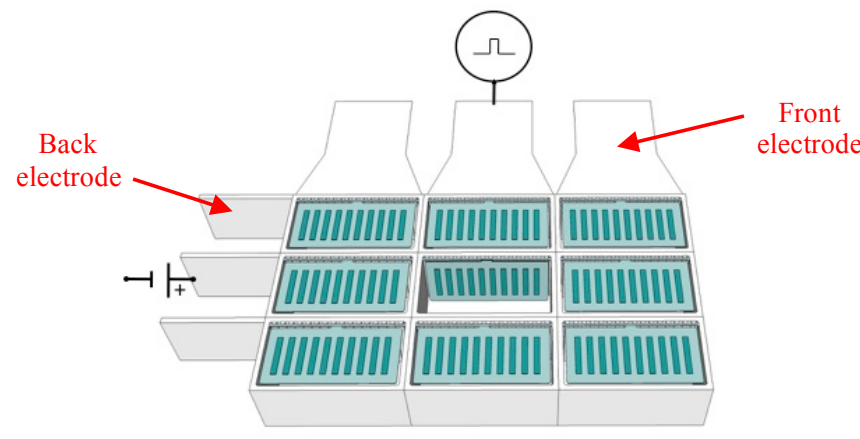

Figure 4: An illustration of how a microshutter is individually actuated: a pulsed DC voltage is applied to the front electrodes (top), and a DC voltage is applied to the back electrodes (left). The front electrodes run vertically while the back electrode runs horizontally.

\section{FABRICATION PROCESS}

For the MSA fabrication, first, we thermally grew a thin layer of silicon oxide on a silicon-on-insulator (SOI) wafer; the buried silicon oxide, device, and handle silicon layers were $3000 \AA, 100$ $\mu \mathrm{m}$, and $350 \mu \mathrm{m}$ thick, respectively. This silicon oxide was used as an etch stop during a silicon etch in a deep reactive etching system (DRIE). Then, a layer of low pressure chemical vapor deposition (LPCVD) silicon nitride was deposited; the stress was between $80-$ $150 \mathrm{MPa}$ (tensile).

A layer of aluminum was deposited in an e-beam deposition system. Then, the aluminum was etched in an aluminum etchant (FujiFilm E-6) at $45^{\circ} \mathrm{C}$ for the front electrodes. The columns of the aluminum electrodes were patterned and separated by $2 \mu \mathrm{m}$ gap in aluminum. The silicon nitride was etched in a reactive ion etch (RIE) system with tetrafluoromethane $\left(\mathrm{CF}_{4}\right)$ to form the microshutters. A layer of molybdenum nitride was deposited in a sputter deposition system to make the microshutters flat. The compressive stress of the film was between $200-400 \mathrm{MPa}$. The film was photo-lithographically patterned in strips and etched in a diluted nitric acid (deionized water: nitric acid $=1: 1$ ) at room temperature. Even though the stress of the aluminum was only around $50 \mathrm{MPa}$ (compressive), there was only slight bowing on the microshutters. However, we observed twisting of the microshutters. Thus, the strips of the molybdenum nitride corrected the twisting and made the bilayer flat. Also, the molybdenum nitride was used as bonding pads in one of our packaging steps: the indium flip-chip bonding.

After the front side processing was complete, the wafer was temporarily bonded to a Pyrex wafer using an acetone soluble wax (Crystalbond 509); the Pyrex wafer was used to perform a back-tofront photolithography alignment. In order to prevent the microshutters getting stuck to the silicon frames, the tolerance of the back to front alignment had to be within $1.5 \mu \mathrm{m}$. Then, the handle silicon was thinned down to $100 \mu \mathrm{m}$ in a lapping system and then completely removed in a DRIE system to expose the buried oxide.

On the back side, the buried silicon oxide was first photolithographically patterned and dry etched in a RIE system using $\mathrm{CF}_{4}$. The buried silicon oxide was used to create $6 \mu \mathrm{m}$ deep and $20 \mu \mathrm{m}$ wide silicon trenches in the $9 \mu \mathrm{m}$ wide silicon frame. The silicon frame is first etched in a deep reactive etching system (DRIE) as shown in the step 1 of Figure 5. Then, the photoresist used for the DRIE etch mask was stripped, and the previously patterned silicon oxide was used as a hard mask to etch the silicon trenches $6 \mu \mathrm{m}$ deep. The trenches were used to separate back electrodes when the subsequent aluminum was deposited at an angle. Figure 6 shows a SEM image of the back side of the MSA and an optical photograph of the separation of the aluminum on bottom of the trench. On the back walls, when the microshutters were held open, we found out that some microshutters were stuck to the smooth walls. Thus, the ribs in the back walls were designed to reduce the microshutter contact surface area and etched to prevent the stiction.

On the back walls, a layer of alumina was deposited conformably using trimethylaluminum (TMA) and oxygen plasma at room temperature in an ALD system to insulate the back side of walls as shown in the step 2 of Figure 5. We needed to keep low temperature because the wafer was still bonded to the Pyrex with the wax, which softens around $70{ }^{\circ} \mathrm{C}$.

On top of the alumina, a layer of aluminum was deposited at an angle in an e-beam deposition system to create the electrodes only on the back walls as shown in the step 3 of Figure 5. On top of the aluminum, a layer of silicon oxide was deposited at a different angle in the same e-beam system as shown in step 4 of Figure 5. The difference in the deposition angles was to cover the aluminum electrode completely with the silicon oxide. The silicon oxide was used as a hard mask for the subsequent dry etch of the alumina in the step 5 of Figure 5. The alumina was only etched behind the microshutter blades with boron trichloride $\left(\mathrm{BCl}_{3}\right)$ in an inductively coupled plasma (ICP) RIE system.

The MSA arrays were first release in acetone and rinsed in methanol, and then all the microshutters were freed by removing the thermal silicon oxide in a vapor HF system; the hard mask of the ebeam deposited silicon oxide was also removed in vapor HF. We found out that the vapor HF does not etch either aluminum or molybdenum nitride. 
Lastly, in order to further prevent the stiction of the microshutters to either the silicon back walls or the aluminum light shields, we deposited a monolayer of a hydrophobic film: ALD perfluorodecyltrichlorosilane (FDTS). We measured the thickness to be $10-20 \AA$, and the water contact angle to be $108^{\circ}$ on monitor silicon wafers.

For the silicon substrate fabrication, we grew a layer of silicon oxide. Then, we deposited a layer of aluminum in an e-beam system and patterned aluminum with the E-6 aluminum etchant. A layer of molybdenum nitride was deposited in a sputter system and pattered in the diluted nitric acid as shown in the step A in Figure 5. The molybdenum nitride was use as the indium bonding pads. A thick layer of indium $\left(\sim 7 \mu \mathrm{m}\right.$ thick, $\left.10 \times 10 \mu \mathrm{m}^{2} \mathrm{big}\right)$ was deposited in a thermal evaporator and lifted off using a thick photoresist (AZ 9245) as a mask as shown in the step B in Figure 5. The thermal silicon oxide was patterned using $\mathrm{CF}_{4}$ in an RIE system with a thick photoreist, and then silicon is etched through as shown in the step C in Figure 5. Figure 7 shows a SEM photo of the patterned indium and optical photos of the patterned aluminum and silicon on the substrate. The silicon openings are smaller than the perimeters of the microshutters to block the light leak. The front sides of the MSA and the silicon substrate are flip-chip bonded using multiple indium bumps per electrical contact pad by applying $90 \mathrm{~N}$ to the entire MSA ( $6 \mathrm{mN}$ per indium bump) at room temperature. The surface of the silicon sustrate was cleaning with forming gas $\left(\mathrm{N}_{2} / \mathrm{H}_{2}\right)$ in a RIE system. When the two layer were pressed by $90 \mathrm{~N}$, the indium bumps were squeezed to $5 \mu \mathrm{m}$ tall. Thus, we had to make sure that there were no particle bigger than $5 \mu \mathrm{m}$ tall on the surface. The aluminum electrodes of the both MSA and substrate run vertically, and they were connected by the indium bumps as shown in the step $\mathrm{D}$ in Figure 5. Thus, the same voltage was applied to the both, and they repel each other. The back side electrdoes on the MSA are wire bonded using aluminum wires to the aluminum pads on the substrate while the front side is connected with indium pads.

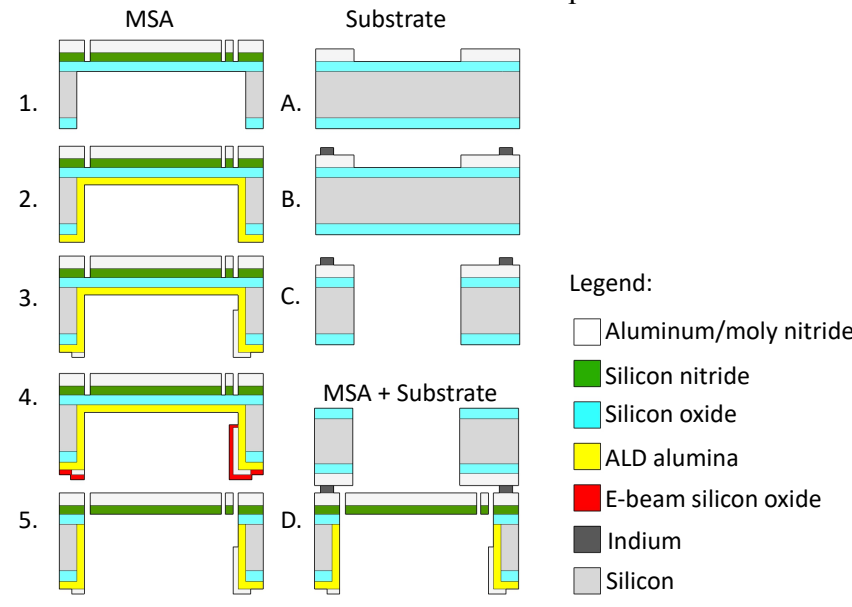

Figure 5: An illustration of the fabrication process of the MSA and the silicon substrate. 1: For the MSA, aluminum, molybdenum nitride, and silicon nitride are patterned on the front side (top). Silicon is etched in a DRIE system from the back side (bottom). 2: ALD aluminum oxide is deposited as an insulator. 3: E-beam aluminum is deposited at an angle for the back side electrodes. 4: E-beam silicon oxide is deposited at an angle. 5: The ALD aluminum oxide is only etched on the bottom in a RIE system, and silicon oxide is removed in vapor $H F$. A: On the silicon substrate, aluminum and molybdenum nitride are deposited and patterned on top of silicon oxide. B: Indium is deposited and lifted off. C: Silicon oxide and silicon are etched. D: Both front sides of the MSA and the substrate are flip-chip bonded.

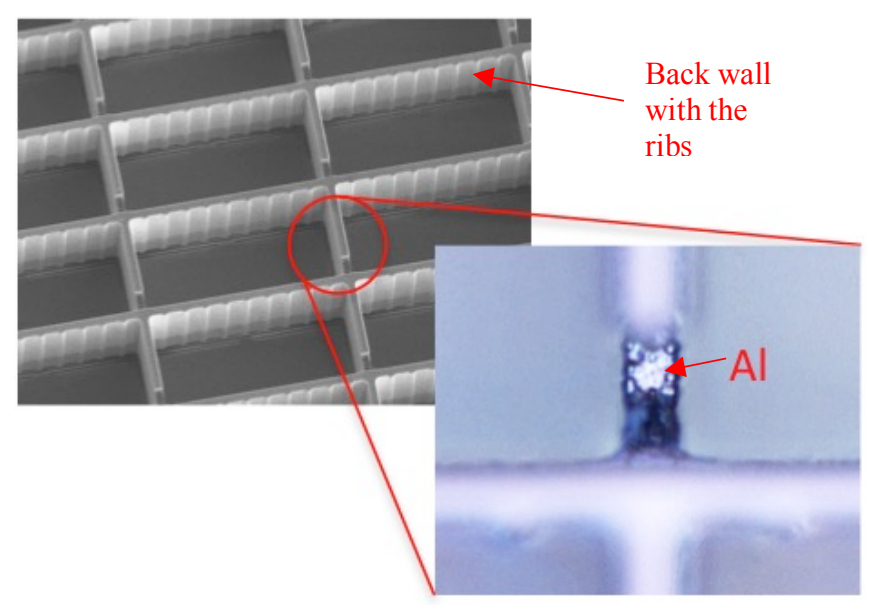

Figure 6: A SEM photo of the back walls with trenches. The closeup photo shows that the aluminum is not continuous on the bottom of the trench. Thus, each back electrode running horizontally is separated.

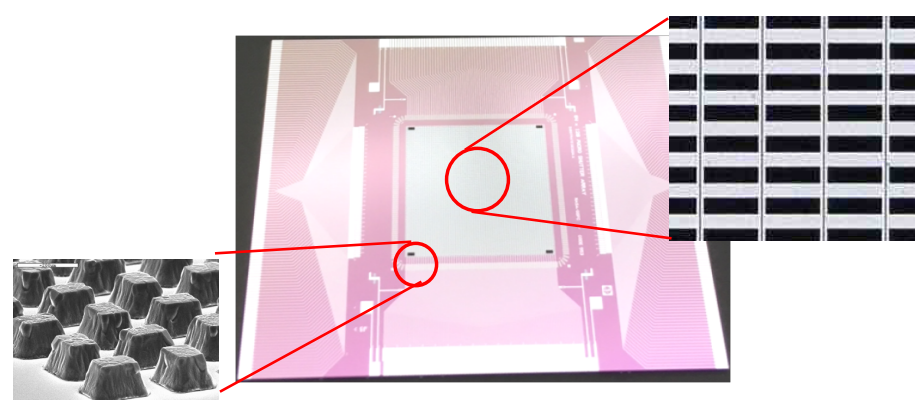

Figure 7: A photo of the silicon substrate with the light shield. The close-up photo (right) with aluminum electrodes are shown. The white columns are the patterned aluminum, and the black is the etched silicon. The close-up photo (left) shows a SEM photo of the indium bumps ( 7 m tall and $10 \times 10 \mu \mathrm{m}^{2} \mathrm{big}$ ) on the molybdenum nitride pads.

\section{DISCUSSION}

In the new back side fabrication process, we utilized the ALD alumina as the insulation layer between the aluminum back electrodes and silicon walls to replace the e-beam deposited alumina that was used in the previous JWST MSA fabrication. The e-beam deposited alumina was not a reliable insulator for the electrostatic actuation of the microshutters; even though the film was $1 \mu \mathrm{m}$ thick, the resistance was in $1-10 \mathrm{M} \Omega$ range, and the breakdown voltage was around $40 \mathrm{~V}$.

Unlike the e-beam deposited alumina, the ALD alumina was very dense and conformal. Thus, the step coverage over the silicon back walls were much better than that of the e-beam alumina. This explains improved the measured resistance of the ALD alumina on the back side of the silicon walls etched in the DRIE step: the resistance was over $1 \mathrm{G} \Omega$. This also increased the breakdown voltages as shown in Figure 8; the breakdown voltage of $1150 \AA$ and $2340 \AA$ thick of ALD alumina were around 60 and $120 \mathrm{~V}$, respectively while $1 \mu \mathrm{m}$ thick e-beam alumina was only around 40 V. 


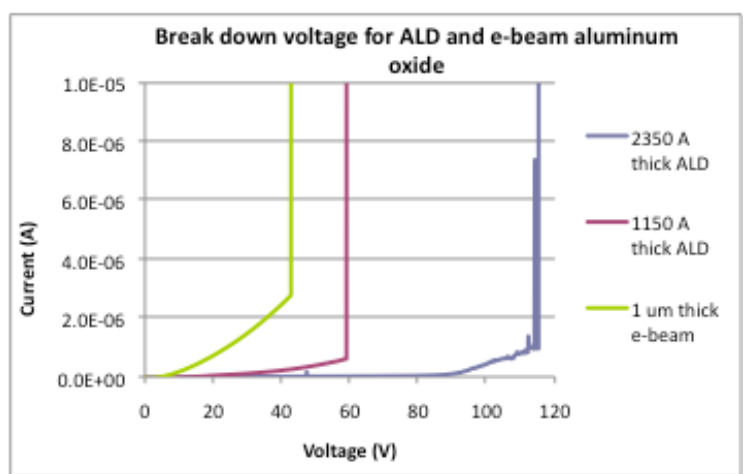

Figure 8: A breakdown voltage comparison by performing I-V curves on $1 \mu \mathrm{m}$ thick e-beam deposited aluminium oxide, 1150 A and 2350 A thick ALD aluminium oxide. The films were deposited on the flat surface of silicon wafers, and $1 \times 1 \mathrm{~mm}^{2}$ of 5000 A thick ebeam aluminium was deposited for this experiment. The breakdown voltage of $1150 A, 2350 A$ thick $A L D$, and $1 \mu \mathrm{m}$ thick e-beam alumina are around $60 \mathrm{~V}, 120 \mathrm{~V}$, and $40 \mathrm{~V}$, respectively.

For the applications in NASA flight missions, we need to keep an operating voltage below $150 \mathrm{~V}$. The JWST MSAs were fabricated on $5000 \AA$ thick silicon nitride. Our finite element analysis model using COMSOL and micro-mechanical force measurements agreed that reducing the silicon nitride from $5000 \AA$ to $2500 \AA$ resulted in the reduction of the stiffness of the silicon nitride by the approximate factor of four [3]. Thus, the microshutters opened with a short DC pulse of $100 \mathrm{~V}$ with 200 microsecond duration to the front electrode. When the microshutters opened with the DC pulse, a DC voltage on the back electrode was applied to bring the microshutters to the back electrode and held the microshutters open. The required back electrode voltage was $-25 \mathrm{~V}$ [4]. During the actuation, the silicon frame on the MSA and silicon substrate are grounded.

The aluminum light shields on the MSA involves 3 lithography steps: a sacrificial photoresist layer, partially exposed light shield bumps $(2500-5000 \AA$ deep $)$ on the sacrificial photoresist, and an aluminum pattern over the sacrificial photoresist. By moving the light shield structure to the silicon substrate, we could save a lot of fabrication time and improve the fabrication yield.

We also fabricated the silicon substrate without the aluminum electrode running parallel to the front electrodes. However, the microshutters never opened; the microshutters were attracted to the silicon frame on the silicon substrate. Thus, we learned that the light shields needed to have aluminum electrodes the same voltage as the front electrodes of the MSA.

\section{RESULTS}

The MSA with the silicon substrate with the light shields was successfully opened and closed. With the actuation scheme discussed above, we were able to open microshutters selectively as shown in Figure 9. Because completing packaging of a PC board and the sub-assembly of the MSA and the substrate with the light shield takes a tremendous effort, these pictures were taken with the MSA without the light shield. The white pixels are where the microshutters were held open while a back illumination was lit; the black background shows that the microshutters stay closed thus block the light.
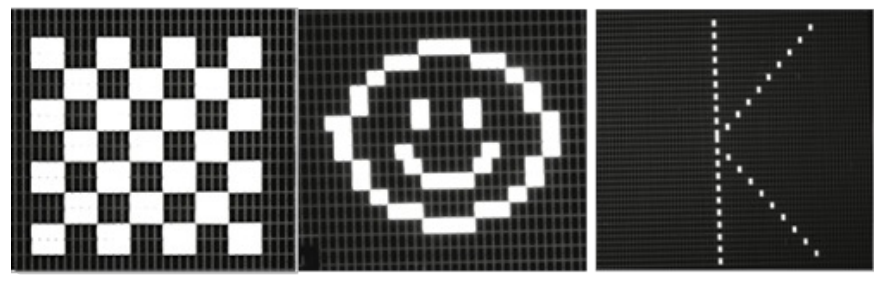

Figure 9. A photo of the microshutters selectively opened with a back light illumination. These photos were taken with the MSA without the light shields. Thus, there were light leaks around the perimeters of the microshutters.

\section{CONCLUSION}

We successfully fabricated the electrostatically actuated microshutter arrays flip-chip bonded to the silicon substrate with the light shields using indium bumps. The array size of the MSA was $64 \times 128$. The microshutters opened with a $100 \mathrm{~V} \mathrm{DC}$ pulse and stayed open with $-25 \mathrm{~V}$ on the back electrode. In order to achieved the electrostatic actuation, we came up with an optimum thickness of silicon nitride. Also, we used the ALD alumina for the insulation on the back electrodes. The use of ALD alumina improved the electrical resistance up to three orders of magnitude and increase the break down voltage. Lastly, the ALD coating of the FDTS film further prevented the stiction of the microshutters to the front and back side.

\section{FUTURE WORK}

For the future NASA flight missions, we are currently developing a big array size of $420 \times 840$ measuring $90 \times 90 \mathrm{~mm}^{2}$ on 6 " wafers. Also, we are redesigning the shapes of the microshutters to optimize the fabrication and the actuation.

So far, all the lithography steps were performed using chromium contact masks in either Suss MA6 or MA8 aligner. Our design rules were limited by how small of a feature we can expose and develop. Also, our fabrication yield was limited to how well we can align in a contact lithography aligner especially the back to front alignment. Currently, we are migrating our alignments and exposures to a Heidelberg direct laser writer. We are expecting to write features smaller than $1 \mu \mathrm{m}$ and improve the front to front and back to front alignments with less than $1 \mu \mathrm{m}$ accuracy. Thus, we are expecting to make much bigger arrays with a higher production yield.

\section{REFERENCES}

[1] M. J. Li, A. D. Brown, A. S. Kutyrev, S. H. Moseley, and V. Mikula, "JWST Microshutter Array System and Beyond," Proc. SPIE 7594, 2010, 1-9.

[2] M. J. Li, T. Adachi, C. A. Allen, S. R. Babu, S. Bajikar, S., et al., "Microshutter Array System for James Webb Space Telescope," Proc. SPIE 6687, 2007, 1-13.

[3] D. E. Burns, J. S. Jones, and M. J. Li, "Mechanical Behavior of Microelectromechanical Microshutters," Proc. SPIE 9170, 2014.

[4] D. E. Burns, L. H. Oh, M. J. Li, D. P. Kelly, A. S. Kutyrev, and S. H. Moseley, "2-D Electrostatic Actuation of Microshutter Arrays," Journal of Microelectromechanical Systems, Vol: PP, Issue: 99, 2015, 1-7.

\section{CONTACT}

*Lance Oh, Lance.H.Oh@nasa.gov 